\title{
A CHARACTERIZATION OF FUNCTION RINGS WITH BOOLEAN DOMAIN
}

\author{
ANDREW B. CARSON
}

(Communicated by Andreas R. Blass)

\begin{abstract}
In $\S 1$ we characterize (effectively in terms of omitted logical types) those countable rings that can be represented as certain specified functions from their Boolean spectra to some member of a universal class of indecomposable rings that has the amalgamation property. In $\S 2$ we show that this characterization fails for uncountable rings and give an alternate (although less interesting) one that does hold for all cardinalities.
\end{abstract}

\section{INTRODUCTION}

In this paper all rings have identity and all morphisms preserve the identity. Results from [1, Theorem 5.3] and [2, Theorem 3.3] show that some commutative semisimple algebraic algebras $R$ (in particular, all countable ones) can be represented as rings of certain continuous functions $X \longrightarrow F$, where $X$ is the Boolean spectrum of $R$ and $F$ is a discretely topologized field. In this paper we characterize all of those rings that can be so represented, where $F$ is now allowed to be any member of a universal class of indecomposable rings that has the amalgamation property. The deeper portion of our sufficient conditions involves the simultaneous omission of a collection of logical types (cf. [7] for background in logic and model theory). (In [3, Chapter 5] an abstract characterization of those rings that are elementarily equivalent to a ring $S$ of certain functions defined on dense open subsets of the Boolean spectrum of $S$ was given. Jech has obtained related results in [9] and [10].)

We shall assume familiarity with the Pierce sheaf (cf. [11] for a detailed treatment and [3, Chapter 1] for a summary) $\mathbf{k}(R)$ of a ring $R$ over $\mathbf{X}(R)$, the Stone space of the Boolean algebra of all central idempotents of $R$. There is a canonical isomorphism $R \cong \Gamma(\mathbf{X}(R), \mathbf{k}(R))$ that represents any ring $R$ as the ring of all global sections of $\mathbf{k}(R)$ over $\mathbf{X}(R)$. For any Boolean space $X$,

Received by the editors September 3,1991 and, in revised form, August 4, 1992.

1991 Mathematics Subject Classification. Primary 16S60; Secondary 03C60. zation.

Key words and phrases. Function rings, Boolean domain, indecomposable range, characteri-

Research for this paper was conducted at the Mathematical Sciences Research Institute (Berkeley) and at the University of Saskatchewan. Research at MSRI supported in part by NSF Grant DMS-8505550. The author was also partially supported by NSERC (Canada) operating grant OGP 0009079. 
discretely topologized ring $F$, and families $\left\{C_{\alpha}: \alpha \in \mathscr{I}\right\}$ and $\left\{F_{\alpha}: \alpha \in \mathscr{I}\right\}$ of closed subsets of $X$ and subrings of $F$, let $\mathscr{C}\left(X, F ;\left\{\left(C_{\alpha}, F_{\alpha}\right): \alpha \in \mathscr{I}\right\}\right)$ denote the ring of all continuous functions $f: X \longrightarrow F$ such that $f\left(C_{\alpha}\right) \subseteq F_{\alpha}$, for all $\alpha \in \mathscr{I}$. If $R$ is commutative and has the above form, then it can be shown that $X=\mathbf{X}(R)$. Suppose that $R$ is a ring, $X=\mathbf{X}(R)$, and is a class of indecomposable rings. Define $R$ to be an $\mathscr{M}$-standard function ring iff it has the above form, for some $F \in \mathscr{M}$. Call $R$ a standard function ring iff it is an $\{F\}$-standard function ring, for some indecomposable ring $F$. In terms of sheaves, $R$ is a standard function ring having the above form iff there is an embedding $\mathbf{k}(R) \subseteq \mathbf{X}(R) \times F$, where $\mathbf{X}(R) \times F$ is the simple sheaf with the product topology. Equivalently, $R$ is an $\mathscr{M}$-standard function ring iff there is an embedding $\Phi: R \longrightarrow \mathscr{C}(\mathbf{X}(R), F)$, for some $F \in \mathscr{M}$, such that $\boldsymbol{\Phi}(\mathbf{B}(R))=\left\{\chi_{C}: C\right.$ is a clopen subset of $\left.\mathbf{X}(R)\right\} . \quad\left(\chi_{C}\right.$ denotes the characteristic function of $C$ throughout this paper. This equivalence occurs in [1, Theorem 5.3], although the setting there is less general.)

In a subsequent paper we shall study twisted as opposed to standard function rings. Such rings $R$ consist of certain twisted functions defined on the Stone space $Y$ of some Boolean algebra $A \supseteq \mathbf{B}(R)$. Both twisted and standard function rings are examples of function rings. A ring $R=\Gamma(\mathbf{X}(R), \mathbf{k}(R))$ is an $\mathscr{M}$-function ring (with domain $Y$ ) iff there is a Boolean algebra $A \supseteq \mathbf{B}(R)$, with Stone space $Y$ and corresponding continuous and onto map $\pi: Y \longrightarrow$ $\mathbf{X}(R)$, and an embedding ${ }^{\wedge}: \Gamma(\mathbf{X}(R), \mathbf{k}(R)) \longrightarrow \mathscr{C}(Y, F)$ such that the map $\mathbf{k}_{x}(R) \longrightarrow F$ given by $r(\pi(y)) \longrightarrow \hat{r}(y)$ is a monomorphism, where $y$ is fixed but arbitrary and $r$ varies over $\Gamma(\mathbf{X}(R), \mathbf{k}(R))$. Clearly, a standard function ring is a function ring, with $Y=\mathbf{X}(R)$, and $\pi=1_{Y}$. At present readers should ignore the details of this strange and technical definition. For the purposes of this paper, we need only consider standard function rings. However, with an eye to applications in [4], we have cast some of the present work generally enough to include those that are not standard.

For any finite subset $F$ of a ring, let $F$ ) denote the subring that it generates. In $\S 1$ we shall show (roughly speaking) that a countable ring $R$ is a standard function ring iff

(for each finite subset $\left.\left\{r_{1}, \ldots, r_{n}\right\} \subseteq \Gamma(\mathbf{X}(R), \mathbf{k}(R))\right) \quad\left\{\left\langle r_{1}(x)\right.\right.$, $\left.\left.\ldots, r_{n}(x)\right\rangle \subseteq \mathbf{k}_{x}(R): x \in \mathbf{X}(R)\right\}$ contains only finitely many rings, up to particular isomorphisms, and certain other minor conditions hold.

Clearly, any standard function ring satisfies $(*)$, as its elements all have finite range and may be identified with the sections appearing in $(*)$. We find it remarkable that little else is required to yield $\mathscr{M}$-standard function rings, in the countable case, provided that the class $\mathscr{M}$ is suitable. In $\S 2$ we show that this characterization fails, for unccuntable rings, although we achieve an alternate characterization holding for all cardinalities, by suitably strengthening $(*)$. The distinction between these two characterizations is evidence that uncountable "functionlike" rings may be very complicated.

Our restrictions on the class $\mathscr{M}$ are necessitated by the nature of our proofs. We asssume that $\mathscr{M}$ is an elementary class, in order to allow model-theoretic arguments. To show that a ring $R$ is an $\mathscr{M}$-standard function ring, we initially show that for each $x \in \mathbf{X}(R)$ there is an embedding $\lambda_{x}: \mathbf{k}_{x}(R) \longrightarrow$ 
$F_{x}$, for some $F_{x} \in \mathscr{M}$. We then amalgamate the $\lambda_{x}$ by obtaining embeddings $\rho_{x}: F_{x} \longrightarrow F$ into some fixed element $F \in \mathscr{M}$ such that the map $\hat{r}: \mathbf{X}(R) \longrightarrow F$ given by $\hat{r}(x)=\rho_{x}\left(\lambda_{x}(r(x))\right)$ is continuous, for each $r \in$ $\Gamma(\mathbf{X}(R), \mathbf{k}(R))$. The required embedding $R \longrightarrow \mathscr{C}(\mathbf{X}(R), F)$ is simply the map $R \cong \Gamma(\mathbf{X}(R), \mathbf{k}(R)) \hat{\longrightarrow} \mathscr{C}(\mathbf{X}(R), F)$. We accomplish this amalgamation by requiring that $\mathscr{M}^{\forall}$, the class of all subrings of $\mathscr{M}$, has the amalgamation property (defined below). Routinely, $\mathscr{M}^{\forall} \supseteq \mathscr{M}$, and $\mathscr{M}^{\forall}$ is a universal class (i.e., $\mathscr{M}^{\forall}$ can be axiomatized using first-order sentences that only involve the quantifier " $\forall$ "; cf. [7, Corollary 3.2.5]). For this reason we may replace $\mathscr{M}$ with $\mathscr{M}^{\forall}$ and add the assumption “ $\mathscr{M}$ is a universal class", without any significant loss of generality.

Definition. A class $\mathcal{N}$ of rings has the amalgamation property iff, whenever $A$, $B$, and $C \in \mathscr{N}$ and $f: A \longrightarrow B$ and $g: A \longrightarrow C$ are embeddings, there exist $D \in \mathscr{N}$ and embeddings $h: B \longrightarrow D$ and $k: C \longrightarrow D$ such that $h f=k g$.

It is well known that $\mathscr{M}^{\forall}$ has the amalgamation property, where $\mathscr{M}$ is the class of all fields or the class of all division rings. (In this context, the class of division rings must be axiomatized in a language including a unary operation symbol ${ }^{-1}$, and the axioms must include $(\forall r)\left(r \neq 0 \Rightarrow r r^{-1}=1\right) \wedge\left(0^{-1}=0\right)$. This guarantees that any substructure of a division ring is also a division ring.)

We are indebted to the referee for pointing out that the amalgamation property for the class $\mathscr{M}$ of possible stalks has played a key role in several other structure results obtained using sheaf representations, such as [8] and [12].

\section{COUNTABLE FUNCTION RINGS}

The following concepts will be used to characterize the countable standard function rings.

Definition 1.1. Suppose that $R$ is a ring and that $\mathscr{M}$ is a class of indecomposable rings. Identify $R=\Gamma(\mathbf{X}(R), \mathbf{k}(R))$. Throughout this definition, $n \in$ $N-\{0\} ; u, v, w, z_{1}, \ldots, z_{n}$ are variables; $\bar{z}=\left(z_{1}, \ldots, z_{n}\right)$; and $\mathscr{F}$ is a finite collection of formulae of the form $\phi(\bar{z})$, each of which is either atomic or is the negation of an atomic formula.

(1) $\operatorname{id}(u)$ is the formula $\left(u^{2}=u\right) \wedge(\forall v)(u v=v u)$.

(2) $R$ has clopen support iff $\{x \in \mathbf{X}(R): r(x) \neq 0\}$ is clopen (closed and open) in $\mathbf{X}(R)$, for all $r \in R$.

(3) $R$ has the $\mathscr{M}$-local joint embedding property (lje-property) iff there exists $F \in \mathscr{M}$ such that, for all $x \in \mathbf{X}(R)$, there is an embedding $\mathbf{k}_{x}(R) \subseteq$ $F$. (By [3, pp. 63-65], the $\mathscr{M}$-lje-property for rings with clopen support is preserved under elementary equivalence.)

(4) $P_{\mathscr{F}}^{1}(w, \bar{z})$ is a formula such that, for all $\bar{r} \in R^{n}$ and $e \in R, R \models$ $P_{\mathscr{F}}^{1}(e, \bar{r})$ iff

(i) $e \in \mathbf{B}(R)-\{0\}$ and

(ii) $\mathbf{k}_{x}(R) \vDash \phi(\bar{r}(x))$ iff $\mathbf{k}_{y}(R) \vDash \phi(\bar{r}(y))$, whenever $\phi(\bar{z}) \in \mathscr{F}$ and $x$ and $y \in \mathbf{X}(R)$ satisfy $e(x)=1$ and $e(y)=1 . \quad(\bar{r}(x)$ abbreviates $\left.r_{1}(x), \ldots, r_{n}(x).\right)$

(5) For each $k \geq 1, P_{\mathscr{F}}^{k}(w, \bar{z})$ is a formula such that whenever $e \in R$ and $\bar{r} \in R^{n}, R \models P_{\mathscr{F}}^{k}(e, \bar{r})$ iff $e \in B(R)-\{0\}$ and, whenever $e_{1}, \ldots, e_{k+1} \in$ 
$\mathbf{B}(R)-\{0\}$ are such that

(i) $R \models P_{\mathscr{F}}^{1}\left(e_{i}, \bar{r}\right) \wedge\left(e_{i} e=e_{i}\right)$, whenever $1 \leq i \leq k+1$, and

(ii) $0=e_{i} e_{j}$, whenever $1 \leq i<j \leq k+1$; then

(iii) $R \models P_{\mathscr{F}}^{1}\left(e_{i}+e_{j}, \bar{r}\right)$, for some $i$ and $j$ such that $1 \leq i<j \leq k+1$.

(6) $R$ is potentially finite-valued (p.f.v.) iff, for all $n \geq 1$ and all $\bar{r}=$ $\left(r_{1}, \ldots, r_{n}\right) \in R^{n}$, there exists $k \geq 1$ such that, for all finite $\mathscr{F}$ as described above, $R \models P_{\mathscr{F}}^{k}(1, \bar{r})$.

Note that each formula of the form $P_{\mathscr{F}}^{k}(w, \bar{z})$ can be viewed as a first-order formula (independent of $R$ ) in the language of rings since, if $e \in \mathbf{B}(R)-\{0\}$, $\bar{r} \in R^{n}$, and $\phi(\bar{z}) \in \mathscr{F},(\alpha)$ and $(\beta)$ are equivalent, where

$(\alpha) \mathbf{k}_{x}(R) \vDash \phi(\bar{r}(x))$ for all $x \in \mathbf{X}(R)$ such that $e(x)=1$ and

( $\beta) \quad R \models(\forall u)[(\operatorname{id}(u) \wedge(0 \neq u=u e)) \Rightarrow \phi(u \bar{r})] . \quad\left(u \bar{r}\right.$ abbreviates $u r_{1}, \ldots$, $\left.u r_{n}.\right)$

To establish this first show that, if $\phi$ is atomic, then $(\alpha)$ is equivalent to

$$
R \models \phi(e \bar{r}) \text {. }
$$

Of course $(\beta)$ and $(\gamma)$ are simply special cases of sheaf-theoretic forcing-a tool that has already found wide application. Note that $R$ has clopen support iff (for each $r \in R$ ) there exists $e \in \mathbf{B}(R)$ such that $r e=r$ and, whenever $f \in \mathbf{B}(R)$ is such that $r f=0$, ef $=0$.

The concept of a p.f.v. ring can be alternatively formulated as follows, for rings with clopen support: For each $n \geq 1$ and $\bar{r}=\left(r_{1}, \ldots, r_{n}\right) \in R^{n}$, define the relation $\sim_{F}$ on $\mathbf{X}(R)$ by $x \sim_{F} y$ iff the map $r(x) \rightarrow r(y)$ (where $r$ varies over $\left\langle\left\{r_{1}, \ldots, r_{n}\right\}\right\rangle$ and we identify $\left.R=\Gamma(\mathbf{X}(R), \mathbf{k}(R))\right)$ is a well-defined isomorphism between the obvious subrings of $\mathbf{k}_{x}(R)$ and $\mathbf{k}_{y}(R)$, respectively. Then $R$ is p.f.v. iff, for each $n \geq 1$ and $\bar{r} \in R^{n}$, the equivalence classes of $\sim_{F}$ form a finite partition of $\mathbf{X}(R)$ into clopen sets.

The next definition and result should clarify these concepts.

Definition 1.2. Let $\mathscr{M}$ be a universal class of indecomposable rings that has the amalgamation property. Then $R$ is an $\omega$-abstract $\mathscr{M}$-standard function ring iff it is a ring, has clopen support, is potentially finite valued, and has the $\mathscr{M}$-lje-property.

Proposition 1.3. Let $\mathscr{M}$ be as in Definition 1.2. Then:

(1) If $R$ is an $\mathscr{M}$-function ring, then $R$ is an $\omega$-abstract $\mathscr{M}$-standard function ring.

(2) Any $\mathscr{M}$-standard function ring is an $\omega$-abstract $\mathscr{M}$-standard function ring.

Proof. (1) Let $R$ be a function ring, and identify $R=\Gamma(\mathbf{X}(R), \mathbf{k}(R))$. By definition we have a Boolean algebra $A \supseteq \mathbf{B}(R)$ with Stone space $Y$ and a continuous onto map $\pi: Y \longrightarrow \mathbf{X}(R), F \in \mathscr{M}$, and a monomorphism ' : $R \longrightarrow \mathscr{C}(Y, F)$ such that, for each $y \in Y$, the map $\mathbf{k}_{\pi(y)}(R) \longrightarrow F$ given by $r(\pi(y)) \longrightarrow \hat{r}(y)$ is an embedding. Thus $R$ has the $\mathscr{M}$-lje-property.

To see that $R$ has clopen support, fix $r \in R$ and let $C=\{y \in Y: \hat{r}(y) \neq 0\}$. As elements of $\mathscr{C}(Y, F)$ are locally constant, both $C$ and $Y-C$ are clopen in $Y$ and thus are compact. Thus $\pi(C)$ and $\pi(Y-C)$ are compact and thus closed. By our hypothesis on ' (regarding the maps $\mathbf{k}_{\pi(y)}(R) \longrightarrow F$ ), 
$\pi(C)$ and $\pi(Y-C)$ are complements in $X$ and thus are clopen. Finally, use the identification $R=\Gamma(\mathbf{X}(R), \mathbf{k}(R))$ and the hypothesis on ' to show that $\{x \in \mathbf{X}(R): r(x) \neq 0\}=\pi(C)$.

To show that $R$ is p.f.v., choose, for each $x \in \mathbf{X}(R), y_{x} \in Y$ such that $\pi\left(y_{x}\right)=x$ and define an embedding ${ }^{*}: R \longrightarrow F^{\mathbf{X}(R)}$ by $r^{*}(x)=\hat{r}\left(y_{x}\right)$. Now let $n \geq 1, \bar{r}=\left(r_{1}, \ldots, r_{n}\right) \in R^{n}$, and $\mathscr{F}$ be arbitrary as in Definition 1.1 . As $Y$ is compact and each $\hat{r}_{i}$ (not $r_{i}^{*}$ ) is continuous, each $\hat{r}_{i}$ (and hence each $r_{i}^{*}$ ) has finite range. Consequently there exist $n_{\vec{r}} \in N$ independent of $\mathscr{F}$ and a partition $\left\{C_{1}, \ldots, C_{n_{r}}\right\}$ of $\mathbf{X}(R)$ into (not necessarily clopen) sets on which each $r_{i}^{*}$ is constant. We shall now show that $R \models P_{\mathscr{F}}^{n_{r}}(1, \bar{r})$. For each $x \in \mathbf{X}(R)$ let $N_{x}=\left\{y \in \mathbf{X}(R): \mathbf{k}_{x}(R) \models \phi(\bar{r}(x))\right.$ iff $\mathbf{k}_{y}(R) \models \phi(\bar{r}(y))$, for all $\left.\phi(\bar{z}) \in \mathscr{F}\right\}$. Each $N_{x}$ is clopen in $\mathbf{X}(R)$, as elements of $R$ have clopen support, and $\mathscr{F}$ is a finite collection of formulae of the form $\phi(\bar{z})$, where $\phi$ is either atomic or is the negation of an atomic formula. By definition, $\left\{N_{x}: x \in \mathbf{X}(R)\right\}$ is a partition of $\mathbf{X}(R)$ and $x \in N_{x}$ (for all $x \in \mathbf{X}(R)$ ), although there will usually exist distinct $x$ and $y$ such that $N_{x}=N_{y}$. By the definition of ${ }^{*}: R \longrightarrow F^{\mathbf{X}(R)}$ (and the fact that the above maps $\mathbf{k}_{\pi(y)}(R) \longrightarrow F$ are monomorphisms), we also have $N_{x}=\left\{y \in \mathbf{X}(R): F \models \phi\left(r_{1}^{*}(x), \ldots, r_{n}^{*}(x)\right)\right.$ iff $F \models \phi\left(r_{1}^{*}(y), \ldots, r_{n}^{*}(y)\right)$, for all $\phi \in \mathscr{F}\}$. Thus $C_{i} \cap N_{x}=\varnothing$ or $C_{i} \subseteq N_{x}$, whenever $1 \leq i \leq n_{\bar{r}}$ and $x \in \mathbf{X}(R)$. Thus there exist $m \leq n_{\bar{r}}$ and $x_{1}, \ldots, x_{m} \in \mathbf{X}(R)$ such that $\left\{N_{x_{1}}, \ldots, N_{x_{m}}\right\}$ is a partition of $\mathbf{X}(R)$, and there exists $j_{i}$ such that $C_{j_{i}} \subseteq N_{x_{i}}$, whenever $1 \leq i \leq m$. The definition of the $N_{x}$ now yields $R \models P_{\mathscr{F}}^{m}(1, \bar{r})$, so $R \models P_{\mathscr{F}}^{n_{r}}(1, \bar{r})$. Hence (1) holds.

Conclusion (2) is a special case of (1).

The main result of this section states that the converse of Proposition 1.3(2) holds, when $R$ is countable.

Theorem 1.4. Let $R$ be a countable ring and $\mathscr{M}$ a universal class of indecomposable rings that has the amalgamation property. Then $R$ is an $\mathscr{M}$-standard function ring iff it is an $\omega$-abstract $\mathscr{M}$-standard function ring.

Proof. Suppose that $R$ is an $\omega$-abstract $\mathscr{M}$-standard function ring. Let $\mathscr{A} t_{n}(\bar{z})$ be the class of all formulae of the form $\phi(\bar{z})$, where $\bar{z}=\left(z_{1}, \ldots, z_{n}\right)$ and $\phi$ is either atomic or is the negation of an atomic formula. Let $\mathscr{A} t=\bigcup_{n \geq 1} \mathscr{A} t_{n}(\bar{z})$. Let $R=\left\{r_{n}: n \geq 1\right\}$ be an enumeration of $R$ such that $r_{1}=1$, and add $\left\{c_{r, x}: r \in R\right.$ and $\left.x \in \mathbf{X}(R)\right\}$, a set of new constant symbols, to the first-order language of rings. Let $R_{n}$ be the subring of $R$ generated by $\left\{r_{i}: 1 \leq i \leq n\right\}$ for each $n \geq 1$, identify $R=\Gamma(\mathbf{X}(R), \mathbf{k}(R))$, and let $k^{n}=\left\{r(x): r \in R_{n}\right.$ and $x \in \mathbf{X}(R)\}$. (Warning: We will likely have $\mathbf{B}\left(R_{n}\right) \neq \mathbf{B}(R), \mathbf{X}\left(R_{n}\right) \neq \mathbf{X}(R)$, and $k^{n} \neq \mathbf{k}\left(R_{n}\right)$.) Fix $n \geq 1$, let $\bar{r}=\left(r_{1}, \ldots, r_{n}\right)$ and $\bar{z}=\left(z_{1}, \ldots, z_{n}\right)$, and choose the least $m_{n}$ such that $R \models P_{\mathscr{F}}^{m_{n}}(1, \bar{r})$ for all finite $\mathscr{F} \subseteq \mathscr{A} t_{n}(\bar{z})$. (Such an $m_{n}$ exists because $R$ is p.f.v.) Thus there exist finite $\mathscr{F} \subseteq \mathscr{A} t_{n}(\bar{z})$ and a partition $\left\{U_{n, 1}, \ldots, U_{n, m_{n}}\right\}$ of $\mathbf{X}(R)$ into nonempty clopen sets such that, for all $\phi \in \mathscr{F}, x, y \in U_{n, i}$, and $x^{\prime} \in U_{n, j}$ (where $i, j \in\left\{1, \ldots, m_{n}\right\}$ and $i \neq j)$,

(a) $\mathbf{k}_{x}(R) \models \phi(\bar{r}(x))$ iff $\mathbf{k}_{y}(R) \models \phi(\bar{r}(y))$, and

(b) there exists $\lambda(\bar{z}) \in \mathscr{F}$ such that $\mathbf{k}_{x}(R) \vDash \lambda(\bar{r}(x))$ iff $\mathbf{k}_{x^{\prime}}(R) \vDash$ $\neg \lambda\left(\bar{r}\left(x^{\prime}\right)\right)$.

Note that for all finite $\mathscr{G} \subseteq \mathscr{A} t_{n}(\bar{z})$ we also have $R \vDash P_{\mathscr{F} \cup \mathscr{G}}^{m_{n}}(1, \bar{r})$, so there 
exists a partition $\left\{V_{1}, \ldots, V_{m_{n}}\right\}$ of $\mathbf{X}(R)$ into nonempty clopen sets such that (a) holds whenever $\phi(\bar{z}) \in \mathscr{F} \cup \mathscr{G}, 1 \leq i \leq m_{n}$, and $x, y \in V_{i}$. However, in view of (b), $\left\{V_{1}, \ldots, V_{m_{n}}\right\}$ must be a refinement of $\left\{U_{n, 1}, \ldots, U_{n, m_{n}}\right\}$ so that, after suitable reindexing, $U_{n, i}=V_{i}$, whenever $1 \leq i \leq m_{n}$. Thus (as $\mathscr{G}$ is arbitrary):

(c) Assertion (a) holds for all $\phi(\bar{z}) \in \mathscr{A} t_{n}(\bar{z})$, whenever $x$ and $y$ belong to some common $U_{n, i}$.

By (c) we have:

(d) The map $r(x) \longrightarrow r(y)$ is an isomorphism $k_{x}^{n} \cong k_{y}^{n}$, where $r$ varies over $R_{n}$ and $x, y \in U_{n, i}$ for some $i \in\left\{1, \ldots, m_{n}\right\}$.

A similar application of (b) yields:

(e) $\left\{U_{n_{1}, 1}, \ldots, U_{n_{1}, m_{n_{1}}}\right\}$ is a refinement of $\left\{U_{n, 1}, \ldots, U_{n, m_{n}}\right\}$, whenever $1 \leq n \leq n_{1}$.

Since $R$ has the $\mathscr{M}$-lje-property, $1(x)$ and $1(y)$ generate isomorphic subrings of $\mathbf{k}_{x}(R)$ and $\mathbf{k}_{y}(R)$ respectively, for all $x, y \in \mathbf{X}(R)$. Consequently, as $r_{1}=1, R \models P_{\mathscr{F}}^{1}\left(1, r_{1}\right)$, for all finite $\mathscr{F} \subseteq \mathscr{A} t_{1}\left(z_{1}\right)$, so:

(f) $m_{1}=1$ and $U_{1,1}=\mathbf{X}(R)$.

We shall now construct a theory $\mathscr{R}$ such that, whenever the constants $c_{r, x}$ are interpreted in a model $F$ of $\mathscr{R}, F \in \mathscr{M}$, and the map ${ }^{\wedge}: R \longrightarrow F^{\mathbf{X}(R)}$, given by $\hat{r}(x)=c_{r, x}$, is actually an embedding ${ }^{\wedge}: R \longrightarrow \mathscr{C}(\mathbf{X}(R), F)$ such that $\widehat{\mathbf{B}(R)}=\left\{\chi_{C}: C\right.$ is clopen in $\left.\mathbf{X}(R)\right\}$. By $\S 0$ this will establish that $R$ is a standard function ring. Let $\mathscr{R}=\mathscr{R}_{1} \cup \mathscr{R}_{2} \cup \mathscr{R}_{3}$, where $\mathscr{R}_{1}$ is a set of axioms for $\mathscr{M}, \mathscr{R}_{2}=\left\{\phi\left(c_{r_{1}, x}, \ldots, c_{r_{n}, x}\right): n \geq 1, \phi \in \mathscr{A} t_{n}(\bar{z}), x \in \mathbf{X}(R)\right.$, and $\left.\mathbf{k}_{x}(R) \models \phi\left(r_{1}(x), \ldots, r_{n}(x)\right)\right\}$, and $\mathscr{R}_{3}=\left\{c_{r, x}=c_{r, y}: n \geq 1, r \in R_{n}\right.$, and $x, y \in U_{n, i}$, for some $\left.i \in\left\{1, \ldots, m_{n}\right\}\right\}$.

We shall now show that $\mathscr{R}$ is consistent. Let $\mathscr{R}_{\mathscr{F}}$ be a finite subset of $\mathscr{R}$, and choose $n \geq 1$ such that all constants from $\mathscr{R}_{\mathscr{F}}$ have the form $c_{r, x}$, where $r \in R_{n}$ and $x \in \mathbf{X}(R)$. Let $\mathscr{U}=\left\{U_{1,1}, U_{2,1}, \ldots, U_{2, m_{2}}, \ldots, U_{n, m_{1}}, \ldots\right.$, $\left.U_{n, m_{n}}\right\}$. For each $U \in \mathscr{U}$, say $U=U_{n^{\prime}, m^{\prime}}$, pick $x_{U} \in U$, and let $\mathbf{k}_{U}=\mathbf{k}_{x_{U}}^{n^{\prime}}$. Note that whenever $V \subseteq U$ and $V$ and $U \in \mathscr{U},(\mathrm{d})$ and (e) give us an embedding $\mathbf{k}_{U} \longrightarrow \mathbf{k}_{V}$. By (e) and (f) the diagram $\left\{\mathbf{k}_{U} \longrightarrow \mathbf{k}_{V}: U, V \in \mathscr{U}\right.$ and $V \subseteq U\}$ is actually a tree with $\mathbf{k}_{U_{1,1}}$ as its root. Moreover this diagram commutes. The nodes of this tree all belong to $\mathscr{M}\left(=\mathscr{M}^{\forall}\right)$, as $R$ has the $\mathscr{M}$ lje-property. The amalgamation property for $\mathscr{M}$ can now be used repeatedly to obtain $G \in \mathscr{M}$ and an embedding $\mathbf{k}_{U} \longrightarrow G$, for each $U \in \mathscr{U}$, such that the diagram $\left\{\mathbf{k}_{U} \longrightarrow \mathbf{k}_{V}: U, V \in \mathscr{U}\right.$, and $\left.V \subseteq U\right\} \cup\left\{\mathbf{k}_{U} \longrightarrow G: U \in \mathscr{U}\right\}$ commutes. Interpret each constant $c_{r, x}$ from $\mathscr{R}_{\mathscr{F}}$ as follows: Choose $U \in \mathscr{U}$ such that $x \in U$ and $r(x) \in \mathbf{k}_{U}$ (i.e., $x \in U$ and $r \in R_{n^{\prime}}$, where $U=U_{n^{\prime}, m^{\prime}}$, for some $m^{\prime}$ ). Interpret $c_{r, x}$ as the image of $r(x)$ under the map $\mathbf{k}_{x}^{n^{\prime}} \cong \mathbf{k}_{U} \longrightarrow$ $G$. Although there may be several $U \in \mathscr{U}$ such that $x \in U$ and $r(x) \in \mathbf{k}_{U}$, the commutativity of the above diagram indicates that the interpretation of $c_{r, x}$ is independant of which one is used. It is easy to verify that $G$ is a model of $\mathscr{R}_{\mathscr{F}}$. The consistency of $\mathscr{R}$ now follows from the logical compactness principle.

Let $F$ be any model of $\mathscr{R}$, and define ' as above. Trivially $F \in \mathscr{M}$ as $\mathscr{R}_{1} \subseteq \mathscr{R}$. The axioms from $\mathscr{R}_{2}$ ensure that ${ }^{\wedge}: R \longrightarrow F^{\mathbf{X}(R)}$ is a well-defined ring monomorphism. Recall that $\mathbf{B}(R)=\left\{\chi_{C}: C\right.$ is clopen in $\left.\mathbf{X}(R)\right\}$, where 
$\chi_{C}$ (as is appropriate) can be viewed as either the characteristic section of $C$ in $\Gamma(\mathbf{X}(R), \mathbf{k}(R))=R$ or as the characteristic function of $C$ in $F^{\mathbf{X}(R)}$. As $\mathscr{R}_{2} \subseteq \mathscr{R}$, we have $\hat{\chi}_{c}=\chi_{c}$, so $\widehat{B(R)}=\left\{\chi_{C}: C\right.$ is a clopen subset of $\left.\mathbf{X}(R)\right\}$. Finally, as $\mathscr{R}_{3} \subseteq \mathscr{R},\left.\hat{r}_{n}\right|_{U_{n, i}}$ is constant, whenever $n \geq 1$ and $1 \leq i \leq m_{n}$. Thus $\hat{r}_{n}$ is continuous for each $n \geq 1$, so ' $: R \longrightarrow \mathscr{C}(\mathbf{X}(R), F)$ is a ring monomorphism. Hence $R$ is a standard function ring.

The converse was established in Proposition 1.3(2).

\section{UNCOUNTABLE FUNCTIONLIKE RINGS}

By results from [4] a ring $R$ is the ring of all suitably twisted functions on a Boolean space $Y$ iff it is a function ring with domain $Y$. By [2, Theorem 3.4] any commutative semisimple algebraic algebra $R$ over a field is von Neumann regular and is a function ring with domain $Y$, where $Y$ is the Stone space of the Boolean completion of $\mathbf{B}(R)$. By Proposition 1.3(2), $R$ is an $\omega$-abstract standard function ring. However, an example from [1, p. 477] shows that $R$ need not be a standard function ring. Thus the countability condition cannot be omitted from Theorem 1.4. Nonetheless, these comments suggest (falsely, as Theorem 2.1 shows even when $R$ is commutative and regular) that any $\omega$ abstract standard function ring $R$ should be a function ring or at least have an embedding of the form $R \longrightarrow \mathscr{C}(Y, F)$. Theorem 2.1 shows that the structure of uncountable commutative regular algebras containing transcendental elements may be enormously complicated, even when they are $\omega$-abstract standard function rings. (Nonetheless, [3, Chapter 5] shows that any commutative regular algebra is a ring of certain continuous functions defined on dense open subsets of its Boolean spectrum.)

Historically, regular algebras have been a fruitful source of objects that are not function rings. However, the previous examples known to the author (e.g., $[1$, p. 467; 3, Example 5.33; 5, Theorem 8; 6, Theorem A]) are not p.f.v.

Following Theorem 2.1 we will give a characterization of all standard function rings, including the uncountable ones.

Theorem 2.1. Let $\mathscr{M}$ be the class of fields. There exists an $\omega$-abstract $\mathscr{M}$ standard function ring $R$ that cannot be embedded in any ring of the form $\mathscr{C}(Y, G)$, where $G \in \mathscr{M}^{\forall}$ and $Y$ is a compact topological space. Moreover $R$ can be chosen to be a commutative von Neumann regular ring that is an algebra over some field.

Proof. Let $F$ be any field having a countably infinite transcendance basis $T$ over its prime subfield. Choose a partial order $<$ on $T$ such that:

(i) $\langle T,\langle\rangle$ is a tree in which each branch is a copy of $\omega$, and

(ii) if $t \in T$ has height $n$, then $\operatorname{Succ}(t)$ (the set of successors of $t$ ) has exactly $n+2$ elements, denoted $t_{0}, \ldots, t_{n+1}$.

The branches of $T$ are in one-to-one correspondence with the functions $f \in T^{N}$ such that

$f(0)$ is the root of $T$ and $f(n+1) \in \operatorname{Succ}(f(n))$, whenever

$n \geq 0$ and $n+1 \in \operatorname{domain}(f)$.

(Such an $f$ corresponds to the branch range $(f) \subseteq T$, and a branch $B$ corresponds to the function $f$ such that $f(n)$ is the unique element of $B$ having 
height $n$.) Let $\mathscr{B}=\left\{f \in T^{N}: f\right.$ satisfies $\left.(*)\right\}$, and, for the rest of this example, let "branch" mean "an element of $\mathscr{B}$ ". We shall construct branches in stages, using partial branches. For each $n \geq 0$, the set of partial branches of height $n$ is $\mathscr{B}_{n}=\left\{f \in T^{\{0, \ldots, n\}}: f\right.$ satisfies $\left.(*)\right\}$. Whenever $f \in T^{N}$, we clearly have $f \in \mathscr{B}$ iff (for all $n \in N$ ) $\left.f\right|_{\{0, \ldots, n\}} \in \mathscr{B}_{n}$.

Whenever $n \in N, 0 \leq m \leq n+1$, and $f \in \mathscr{B}_{n}$, define $\delta_{f}^{m} \in \mathscr{B}_{n+1}$ by

$$
\delta_{f}^{m}(x)= \begin{cases}f(x) & \text { when } 0 \leq x \leq n, \\ t_{m} & \text { when } x=n+1 \text { and } t=f(n) .\end{cases}
$$

Let $R$ be the subring of $F^{N}$ generated by $\mathscr{B} \cup\left\{\chi_{C}: C \subseteq N\right\}$. Routinely, $\mathbf{X}(R)$ is the Stone-Čzech compactification of $N, \mathbf{k}_{n}(R) \cong\{r(n): r \in R\} \subseteq F$ (for all $n \in N$ ), and $R$ has clopen support.

We shall now show that $R$ has the $\mathscr{M}$-lje-property. Let $\phi(\bar{z})$ be a quantifier free primitive formula in $\bar{z}=z_{1}, \ldots, z_{m}$ (where $m$ depends on $\phi$ ), let $\bar{r}=\left(r_{1}, \ldots, r_{m}\right) \in R^{m}$ and $x \in \mathbf{X}(R)$, and temporarily view each $r_{i}$ as a section $\mathbf{X}(R) \longrightarrow \mathbf{k}(R)$, rather than as a function $N \longrightarrow F$. Thus $\bar{r}(y)=\left(r_{1}(y), \ldots, r_{m}(y)\right) \in\left(\mathbf{k}(R)_{y}\right)^{m}$, whenever $y \in \mathbf{X}(R)$. Suppose that $\mathbf{k}_{x}(R) \models \phi(\bar{r}(x))$. As $N$ is dense in $\mathbf{X}(R)$ and $R$ has clopen support, there exists $n \in N$ such that $\mathbf{k}_{n}(R) \models \phi(\bar{r}(n))$. Since there is an embedding $\mathbf{k}_{n}(R) \subseteq F$, we see that any finite subset of the model-theoretic diagram of $\mathbf{k}_{x}(R)$ can be interpreted in $F$. Logical compactness now yields a field $G$ such that $\mathbf{k}_{x}(R) \subseteq G$.

For the rest of this proof $f(\bar{Z})$ is always a polynomial in $Z_{1}, \ldots, Z_{n^{\prime \prime}}$ (where $n^{\prime \prime}$ depends on $f$ ) over the prime subfield of $F, \bar{r}=\left(r_{1}, \ldots, r_{n^{\prime \prime}}\right) \in$ $\mathscr{B}^{n^{\prime \prime}}$, and $\bar{s}$ and $\bar{t}$ always denote elements of $T^{n^{\prime \prime}}$. Thus $\bar{r}(x)=\left(r_{1}(x), \ldots\right.$, $\left.r_{n^{\prime \prime}}(x)\right) \in T^{n^{\prime \prime}}$, whenever $x \in N$. Let $\operatorname{Repet}(\bar{s})=\left\{(i, j): s_{i}=s_{j}\right\}$. Our proof that $R$ is p.f.v. uses these routine consequences of $T$ being a transcendance base:

(1) If $f(\bar{s})=0$ and $\operatorname{Repet}(\bar{s}) \subseteq \operatorname{Repet}(\bar{t})$, then $f(\bar{t})=0$.

Hence (as $\left.\bar{r} \in \mathscr{B}^{n^{\prime \prime}}\right)$ :

(2) $f(\bar{r}(x))=0$ implies that $f(\bar{r}(y))=0$, whenever $0 \leq y \leq x \in N$.

In contrast to $(1)$ :

(3) If $f(\bar{s}) \neq 0$ and $\operatorname{Repet}(\bar{s}) \supseteq \operatorname{Repet}(\bar{t})$, then $f(\bar{t}) \neq 0$. Thus:

(4) $f(\bar{r}(y)) \neq 0$ implies that $f(\bar{r}(x)) \neq 0$, whenever $0 \leq y \leq x \in N$.

Now fix $\bar{r} \in \mathscr{B}^{n^{\prime \prime}}$ (for some $n^{\prime \prime}$ ), and choose a finite partition $\left\{C_{1}, \ldots, C_{k}\right\}$ of $N$ such that

(5) $\operatorname{Repet}(\bar{r}(x))=\operatorname{Repet}(\bar{r}(y))$, whenever $x$ and $y \in C_{i}$, for some $i \in$ $\{1, \ldots, k\}$. (This is possible with $k \leq n^{\prime \prime}$, as $\bar{r} \in \mathscr{B}^{n^{\prime \prime}}$.)

Thus:

(6) $f(\bar{r}(x))=0$ iff $f(\bar{r}(y))=0$, whenever $x$ and $y \in C_{i}$, for some $i \in$ $\{1, \ldots, k\}$.

Note that each atomic formula $\phi(\bar{z})$ has the form $f(\bar{z})=0$. Thus $R \vDash$ $P_{\mathscr{G}}^{k}(1, \bar{r})$, whenever $\mathscr{G}$ is a finite collection of such formulae and/or the negations of such formulae. Although $\bar{r}$ is arbitrary in $\mathscr{B}^{n^{\prime \prime}}$ rather than in $R^{n^{\prime \prime}}$, 
it follows easily that $R$ is p.f.v. Hence $R$ is an $\omega$-abstract $\mathscr{M}$-standard function ring. Although $R$ need not be von Neumann regular, all of its stalks are integral domains, as it does possess the $\mathscr{M}$-lje-property. We omit the routine proof that $Q_{\mathrm{cl}}(R)$ (the classical quotient ring of $R$ ) also is an $\omega$-abstract $\mathscr{M}$ standard function ring, $\mathbf{X}\left(Q_{\mathrm{cl}}(R)\right)=\mathbf{X}(R), Q_{\mathrm{cl}}(R) \subseteq F^{\mathbf{X}(R)}$, all of the stalks of $\mathbf{k}\left(Q_{\mathrm{cl}}(R)\right)$ are fields, and $Q_{\mathrm{cl}}(R)$ is commutative and von Neumann regular. Clearly $Q_{\mathrm{cl}}(R)$ is an algebra over the rationals. Replace $R$ with $Q_{\mathrm{cl}}(R)$ for the remainder of this proof. We stress that $R \subseteq F^{\mathbf{X}(R)}$ still holds. For each $n \in N$ let $k_{n}=\left\{r(n) \in F: r \in R \subseteq F^{\mathbf{X}(R)}\right\}$ and show that $k_{n} \cong \mathbf{k}_{n}(R)$, so $k_{n}$ is a field.

Suppose (contrary to the theorem) that there is a monomorphism ${ }^{\wedge}: R \longrightarrow$ $\mathscr{C}(Y, G)$ for some $G \in \mathscr{M}^{\forall}$ and compact space $Y$. For each $n \in N$ choose $y_{n} \in Y$ such that $\hat{\chi}_{\{n\}}\left(y_{n}\right)=1_{G}$. (This is possible, as $\hat{\chi}_{\{n\}} \in \mathbf{B}(\mathscr{C}(Y, G))$.) Note that if $r \in R$ and $r(n)=0_{F}$, then $r \cdot \chi_{\{n\}}=0_{R}$, so $\hat{r}\left(y_{n}\right)=\hat{r}\left(y_{n}\right) \cdot 1_{G}=$ $\hat{r}\left(y_{n}\right) \cdot \hat{\chi}_{\{n\}}\left(y_{n}\right)=\left(\widehat{r} \widehat{\chi_{\{n\}}}\right)\left(y_{n}\right)=0_{G}$. Thus the rule $\Phi_{n}(r(n))=\hat{r}\left(y_{n}\right)$ (which clearly preserves + and $\cdot)$ is a well-defined map $\Phi_{n}: k_{n} \longrightarrow G$. It preserves 1 as does. In detail, $\Phi_{n}\left(1_{k_{n}}\right)=\Phi_{n}\left(1_{R}(n)\right)=\left(\hat{1}_{R}\right)\left(y_{n}\right)=1_{\mathscr{C}(Y, G)}\left(y_{n}\right)=1_{G}$. Since $k_{n}$ is a field we see that $\Phi_{n}$ is a monomorphism, for each $n \geq 0$.

We shall define $b \in \mathscr{B} \subseteq R$ such that

(**) The elements of $\left\{\Phi_{i}(b(i)): i \in \operatorname{domain}(b)\right\}$ are all distinct.

This would yield

$$
\left\{\hat{b}\left(y_{i}\right): i \in N\right\} \text { is infinite, }
$$

contrary to the fact that $Y$ is compact and $G$ is discrete, and show that the embedding ${ }^{\wedge}: R \longrightarrow \mathscr{C}(Y, G)$ cannot exist. Suppose that $n \geq 0$ and that $b \in \mathscr{B}_{n}$ has been defined such that $(* *)$ holds. As $\left\{\Phi_{n+1}\left(\delta_{b}^{m}(n+1)\right): 0 \leq m \leq\right.$ $n+1\}$ contains $n+2$ distinct elements, there exists $m \in\{0, \ldots, n+1\}$ such that $\Phi_{n+1}\left(\delta_{b}^{m}(n+1)\right) \notin\left\{\Phi_{i}(b(i)): 0 \leq i \leq n\right\}$. Replace $b$ with $\delta_{b}^{m} \in \mathscr{B}_{n+1}$, and use the equations $\delta_{b}^{m}(i)=b(i)$ for $0 \leq i \leq n$ to see that $(* *)$ still holds. As the new $b \in \mathscr{B}_{n+1}$ is an extension of the original $b \in \mathscr{B}_{n}$, we can continue by induction obtaining $b \in \mathscr{B}$ such that $(* *)$ holds, as was required to establish this theorem.

Remark 2.2. In the above example $\mathbf{B}(R)$ is complete. The following slight modification would yield $\mathbf{B}(R)$ countable yet $R$ still suitable to establish Theorem 2.1: Initially let $R \subseteq F^{N}$ be generated by $\mathscr{B} \cup\left\{\chi_{C}: C\right.$ is a finite or cofinite subset of $N\}$, and then replace $R$ by its classical quotient ring. In this case $\mathbf{X}(R)=N \cup\{\infty\}$, the one-point compactification of the natural numbers, and $\mathbf{k}_{n}(R) \cong F$ for all $n \in N$. In contrast, if $S$ is a (possibly uncountable) commutative semisimple algebraic algebra such that $\mathbf{B}(S)$ is countable or complete, then $S$ is a standard function ring (cf. [2, Theorem 3.3]). Thus in the uncountable case, the $\omega$-abstract standard function rings do not even generalize the semisimple algebraic algebras.

An analysis of the proof of Theorem 1.4 shows that \#(range $\left.\left(r_{i}\right)\right)$, where $r_{i} \in R$, depends upon our choice of indexing for $R$. Specifically, if $r_{1}, \ldots, r_{n} \in$ $R$ and $k$ is the least natural number such that $R \models P_{\mathscr{F}}^{k}\left(1, r_{1}, \ldots, r_{n}\right)$ for all appropriate $\mathscr{F}$, then $\#\left(\operatorname{range}\left(r_{n}\right)\right) \leq k$, even if $R \models P_{\mathscr{G}}^{1}\left(1, r_{n}\right)$, for all appropriate $\mathscr{G}$. If, however, we were to switch $r_{n}$ with $r_{1}$ by reindexing, we would have $\#\left(\operatorname{range}\left(r_{1}\right)\right)=1$. Heuristically, Theorem 1.4 is true because 
countable $R$ can be indexed such that each element $r_{n}$ has only finitely many predecessors, $r_{1}, \ldots, r_{n-1}$. This suggests that the uncountable case might be tackled using the following concept: A ring $R$ is uniformly p.f.v. iff, for each $r \in R$, there exists $n_{r} \geq 1$ such that $R \models P_{\mathscr{F}}^{k}\left(1, r_{1}, \ldots, r_{m}\right)$, whenever $m \geq 1$; $r_{1}, \ldots, r_{m} \in R ; \mathscr{F}$ is appropriate as in Definition 1.1 ; and $k=n_{r_{1}} \cdots n_{r_{m}}$. If $R \subseteq \mathscr{C}(\mathbf{X}(R), F)$ is an $\{F\}$-standard function ring, then $R$ is uniformly p.f.v., with $n_{r}=\#(\operatorname{range}(r))$, for all $r \in R$. On the basis of many examples the author conjectured that any uniformly p.f.v. $\omega$-abstract standard function ring could be represented as a standard function ring in such a way that $\#(\operatorname{range}(r)) \leq n_{r}$, for all $r \in R$. However, this conjecture is false, as the ring $R$ from Theorem 2.1 is uniformly p.f.v. (with $n_{b}=2$ for all $b \in \mathscr{B}$ ) because $k \leq n^{\prime \prime}$ in (5) from the proof. In consequence we believe that a successful formulation of the p.f.v. concept for an uncountable setting must mimic the situation prevailing in standard function rings so strongly that the resultant structure theorems are almost obvious. For the record, we sketch one such approach.

Definition 2.3. A ring $R$ is locally potentially finite valued (1.p.f.v.) iff for each $r \in R$ there exists a finite subset $E(r) \subseteq \mathbf{B}(R)-\{0\}$ such that:

(i) $1=\sum_{e \in E(r)} e$ and $0=f g$, whenever $r \in R$, and $f$ and $g$ are distinct elements of $E(r)$, and

(ii) $R \models P_{\mathscr{F}}^{1}\left(f, r_{1}, \ldots, r_{n}\right)$, whenever $n \geq 1, r_{1}, \ldots, r_{n} \in R, f \neq 0$, and $f$ has the form $f=e_{1} \cdots e_{n}$, where each $e_{i} \in E\left(r_{i}\right)$, and $\mathscr{F}$ is arbitrary as described in Definition 1.1.

Definition 2.4. Let $\mathscr{M}$ be a universal family of indecomposable rings that has the amalgamation property. A ring $R$ is an abstract $\mathscr{M}$-standard function ring iff it is l.p.f.v., has clopen support, and has the $\mathscr{M}$-lje-property.

Theorem 2.5. Let $\mathscr{M}$ be as in Definition 2.4. A ring $R$ is an abstract $\mathscr{M}$ standard function ring iff it is an $\mathscr{M}$-standard function ring.

Proof. Suppose that $R$ is an abstract $\mathscr{M}$-standard function ring. Show that for each $r \in R$ there is a finite partition $\mathscr{P}(r)$ of $\mathbf{X}(R)$ into nonempty clopen subsets such that

$$
\mathbf{k}_{x}(R) \models \phi(\bar{r}(x)) \quad \text { iff } \quad \mathbf{k}_{y}(R) \models \phi(\bar{r}(y)),
$$

whenever $n \geq 1, \bar{r}=\left(r_{1}, \ldots, r_{n}\right) \in R^{n}$, and $x$ and $y \in V$, where $V$ has the form $\varnothing \neq V=V_{1} \cap \cdots \cap V_{n}$, for some $\left(V_{1}, \ldots, V_{n}\right) \in \mathscr{P}\left(r_{1}\right) \times \cdots \times \mathscr{P}\left(r_{n}\right)$, and whenever $\phi$ is either atomic or is the negation of an atomic formula. Let $\left\{c_{r, x}: r \in R\right.$ and $\left.x \in \mathbf{X}(R)\right\}$ be a new set of constant symbols. Let $\mathscr{R}=\mathscr{R}_{1} \cup \mathscr{R}_{2} \cup \mathscr{R}_{3}$, where $\mathscr{R}_{1}$ and $\mathscr{R}_{2}$ are as in the proof of Theorem 1.4 and $\mathscr{R}_{3}=\left\{c_{r, x}=c_{r, y}: r \in R\right.$ and $x$ and $y$ are in the same element of $\mathscr{P}(r)\}$. Show that $\mathscr{R}$ has a model $F$, as $\mathscr{M}$ is a universal class with the amalgamation property and $R$ has the $\mathscr{M}$-lje-property. As in Theorem 1.4 the required embedding ${ }^{\wedge}: R \longrightarrow \mathscr{C}(\mathbf{X}(R), F)$ is given by $\hat{r}(x)=c_{r}, x$.

Conversely, assume that $R \subseteq \mathscr{C}(\mathbf{X}(R), F)$, for some $F \in \mathscr{M}$. For each $r \in R$, let $\mathscr{P}(r)$ be the partition of $\mathbf{X}(R)$ into clopen sets such that $\left.r\right|_{V}$ is constant, for each $V \in \mathscr{P}(r)$. To finish, let $E(r)=\left\{\chi_{V}: V \in \mathscr{P}(r)\right\}$.

While characterizing twisted function rings in [4], we shall determine when a ring $R$ can be nicely embedded in $\mathscr{C}(Y, F)$, where $Y$ might not be $\mathbf{X}(R)$. 
Clearly, the class of $\omega$-abstract standard function rings is closed under elementary equivalence. (Cf. Definitions 1.1 and 1.2 and the intervening remarks.) Thus (despite Theorem 2.1) the Löwenheim-Skolem theorem, Proposition 1.3, and Theorem 1.4 do yield a model-theoretic description of $\omega$-abstract function rings of all cardinalities. Specifically:

Theorem 2.6. Let $\mathscr{M}$ be as in Definition 2.4. Then $R$ has a countable elementary substructure that is an $\mathscr{M}$-standard function ring, in each of the following cases:

(1) $R$ is an $\omega$-abstract $\mathscr{M}$-standard function ring, or

(2) $R$ is an $\mathscr{M}$-function ring.

In contrast:

Proposition 2.7. Let $\mathscr{M}$ be the class of fields. Then there exist $R$ and $S$ such that $R$ is an $\mathscr{M}$-standard function ring, $R \prec S$, yet $S$ is not p.f.v. and is not an $\omega$-abstract $\mathscr{M}^{\forall}$-standard function ring. (Of course $\mathscr{M}^{\forall}$ is the class of integral domains.)

Proof. Let $R=\mathscr{C}(X, F)$, where $X$ is the Stone-Čzech compactification of the natural numbers and $F$ is the field of rational numbers. As in [3, Example 5.33] there exists $S \succ R$ such that $\mathbf{X}(S)=X$, and there exists $s \in \Gamma(\mathbf{X}(S), \mathbf{k}(S))$ such that $s(n)=n \in \mathbf{k}_{n}(S) \cong F$, for all $n \in N \subseteq X$. For each $m \geq 1$ let $\mathscr{F}_{m}=\{(z=1), \ldots,(z=m+1)\}$. Then $S \models \neg P_{\mathscr{F}_{m}}^{m}(1, s)$, so $S$ is not p.f.v.

An additional contrast to our current results occurs in [5]: There is a commutative regular algebra $R$ such that, whenever $R \equiv S, S$ is not an $\mathscr{F}^{\forall}$-standard function ring, where $\mathscr{F}$ is the class of all fields. Applying Theorem 2.6 yields that $S$ is not an $\omega$-abstract $\mathscr{F}^{\forall}$-standard function ring, whenever $R \equiv S$. As noted in [5], this pathology could not occur if $R$ were algebraically closed. (Warning: The phrase "function ring" as used in [5] means "standard function" ring in this paper.)

To summarize this paper, suppose that $R$ has clopen support and has the $\mathscr{M}$-lje-property. Loosely speaking $R$ is an $\mathscr{M}$-standard function ring iff (pretending that it consists of functions) you cannot show that at least one of its elements must have infinite range. This vague principle was given two distinct formulations: Definition 2.4 to classify all standard function rings and Definition 1.2 to classify the countable ones. We regard Definition 1.2 as much weaker than 2.4 and regard Theorem 1.4 as more interesting than 2.5, even though it only applies when $R$ is countable.

Remarks. (1) With minor modifications, the proof of Theorem 1.4 could establish that an $\omega$-abstract $\mathscr{M}$-standard function ring $R$ is an $\mathscr{M}$-standard function ring so long as it has a countable subset $S \subseteq R$ such that $R=\langle S \cup \mathbf{B}(R)\rangle$. In contrast, previous work (cf. [1, 2]) establishing that certain semisimple algebraic algebras are standard function rings only applies when $\mathbf{B}(R)$ is nice (i.e., countable or complete). However, Theorem 1.4 is not (in the strictest sense) a full generalization of this earlier work, as Remark 2.2 shows that it cannot be modified to apply when $\mathbf{B}(R)$ rather than $R$ is countable or when $\mathbf{B}(R)$ is complete.

(2) Our characterization of $\mathscr{M}$-function rings only applies when $\mathscr{M}$ is a universal class of indecomposable rings with the amalgamation property. Recall 
that $\mathscr{R}$, the class of ordered fields, does not have the amalgamation property. To establish this limitation on our results, let $R$ be the ring from [3, Example $5.31^{\prime}$ ] and let $\mathscr{F}$ be the class of all fields. That $R$ is an $\mathscr{F}$-function ring follows from [2], as $R$ is a countable von Neumann regular ring and is an algebraic algebra over some field. Thus the proof of Proposition 1.3 establishes that $R$ is an $\omega$-abstract $\mathscr{F}$-function ring and so is potentially finite valued. Moreover, $R$ has the $\mathscr{R}$-lje-property. Thus $R$ would be an $\omega$-abstract $\mathscr{R}$ function ring, if we allowed that concept to apply despite the fact that $\mathscr{R}$ does not have the amalgamation property. However (cf. [3, p. 92]), $R$ is not an $\mathscr{R}$-function ring.

(3) In contrast to (2) let $X$ the Cantor space and $F$ be the real closure of the rational numbers. Then $\mathscr{C}(X, F)$ is a countable $\mathscr{R}$-function ring that would be an $\omega$-abstract $\mathscr{R}$-function, if we were to allow this terminology to be used with $\mathscr{R}$.

(4) Theorem 2.6 and the second sentence of $\S 2$ yield: Any commutative semisimple algebraic algebra over a field has a countable elementary substructure that is an $\mathscr{F}$-standard function ring. The same conclusion was reached in [5, Theorem 5], under the additional hypothesis that $R$ satisfy some polynomial identity in a single variable.

\section{ACKNOWLEDGMENT}

The author thanks the referee for detecting several misprints in an earlier version of this paper and for suggesting the comments concerning [8] and [12] made at the end of the introduction.

\section{REFERENCES}

1. R. Arens and I. Kaplansky, Topological representations of algebras, Trans. Amer. Math. Soc. 63 (1949), 457-481.

2. A. Carson, Representation of semi-simple algebraic algebras, J. Algebra 24 (1973), 245-257.

3. Res. Notes Math. Ser., vol. 209, Longman Sci. Tech., Essex, 1989.

4. _ _ A characterization of rings of twisted functions, Illinois J. Math. (to appear).

5. _ Rings that are not elementarily equivalent to a function ring, Comm. Algebra 12 (1990), 4225-4234.

6. $\ldots$, Self-injective regular algebras and function rings, Algebra Universalis 29 (1992), 449-454.

7. C. Chang and H. Keisler, Model theory, second ed., Studies in Logic Found. Math., vol. 73, North-Holland, Amsterdam, 1977.

8. S. Comer, Monadic algebras with finite degree, Algebra Universalis 5 (1975), 313-327.

9. T. J. Jech, Abstract theory of abelian operator algebras: an application of forcing, Trans. Amer. Math. Soc. 289 (1985), 133-162.

10. _ Boolean-linear spaces, Adv. Math. 81 (1990), 117-197.

11. R. S. Pierce, Modules over commutative regular rings, Mem. Amer. Math. Soc., vol. 70, Amer. Math. Soc., Providence, RI, 1967.

12. H. Werner, Discriminator algebras. Algebraic representation and model theoretic properties, Akademie Verlag, Berlin, 1978.

Department of Mathematics, University of Saskatchewan, Saskatoon, Saskatchewan, CANADA S7N OWO

E-mail address: carson@math.usask.ca 